\title{
REVIEW
}

\section{Molecular connections between circadian rhythm and genome maintenance pathways}

\author{
Arun Mouli Kolinjivadi', Siao Ting Chong ${ }^{1,2}$ and Joanne Ngeow ${ }^{1,2}$ \\ 1 Lee Kong Chian School of Medicine, Nanyang Technological University Singapore, Singapore, Singapore \\ ${ }^{2}$ Cancer Genetics Service, Division of Medical Oncology, National Cancer Centre Singapore, Singapore, Singapore
}

Correspondence should be addressed to J Ngeow: jongeow@gmail.com

This paper is part of a collection of articles on Circadian Rhythms and Cancer. The guest editors for this section were Joanne Ngeow, David Marc Virshup and Robert Dallman. Joanne Ngeow was not involved in the review process for this paper, on which they are listed as an author.

\begin{abstract}
Co-ordinated oscillation of mammalian circadian clock and cell cycle is essential for cellular and organismal homeostasis. Existing preclinical, epidemiological, molecular and biochemical evidence reveals a robust interplay between circadian clock, genome instability and cancer. Furthermore, recent investigations have demonstrated that the alterations in circadian clock perturb genome stability by modulating the cell-cycle timing, altering DNA replication fork progression, influencing DNA damage response (DDR) and DNA repair efficiency. In this review, we examine the most recent findings from different eukaryotic model systems and discuss the functional interaction between circadian factors with key DNA replication, DDR and DNA repair genes.
\end{abstract}

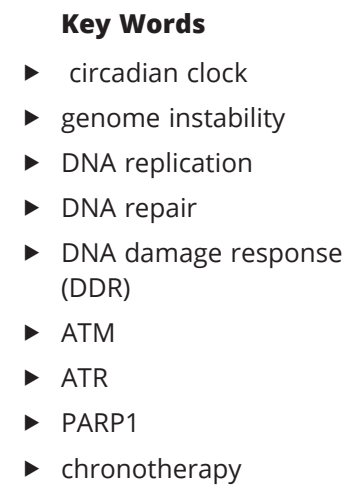

Endocrine-Related Cancer (2020) 28, R55-R66

\section{Introduction}

Generally, many biological processes are spatially and temporally regulated. At the cellular level, starting from the duplication of chromosomal DNA during S (synthesis) phase to the segregation of chromosomes at $\mathrm{M}$ (mitotic) phase of the cell cycle, temporal separation of molecular events is crucial. In a broader perspective, at the organismal level, external environmental cues such as the temperature, light, sleep cycle and nutrient intake influence the biological timing or rhythm that follow a 24-h periodicity governing the essential cellular and molecular events. Several landmark studies in mammals, zebrafish, plants, fungi and cyanobacteria support the concept of biological clock or circadian rhythm impacting different phases of the cell cycle such as DNA synthesis, DNA repair, checkpoint mechanisms and cytokinesis (Mori et al. 1996, Dekens et al. 2003, Matsuo et al. 2003,
Nagoshi et al. 2004, Hirayama et al. 2005, Dong et al. 2010, Masri \& Sassone-Corsi 2010, Kowalska et al. 2013, Laranjeiro et al. 2013, Feillet et al. 2014, Hong et al. 2014). In vertebrates, the timing of embryonic and somatic cell cycle varies widely, and this appears to be governed in part by the rhythmic expression of circadian genes (Ziv \& Gothilf 2006). Altering the duration of cells at different phases of the cell cycle compromises genome stability (Ahuja et al. 2016, Marchal et al. 2019), a hallmark feature of cancer. The biological clock is embedded within every cell as molecular machinery with the capacity to adapt to external changes using their remarkable plasticity feature. Despite the cellular plasticity, persistent changes in the metabolic and environmental cues disturb the biological rhythm resulting in deregulation in cellular and organismal homeostasis. However, the precise mechanism 
by which the circadian clock perturbation results in cancer initiation and progression is far from clear.

In this review article, we aim to discuss the recent findings on how circadian clock perturbation impacts genome stability to trigger tumour initiation and progression. Furthermore, we summarise the most current evidence on how circadian clock genes (CCGs) regulate directly or indirectly the DNA replication programme, the rhythmicity of DNA damage response (DDR) and DNA repair pathway in the presence of exogenous or endogenous DNA damage. Finally, we briefly speculate the potential of using chronotherapy for designing new therapeutic strategies for chromosome instability disorders.

\section{Mammalian circadian clock machinery: transcriptional and its translational regulation}

In vertebrates, the circadian rhythm is governed by the central and peripheral clocks. The central circadian clock is located in the superchiasmatic nucleus (SCN) of the brain, in the hypothalamus, which is normally adjustable by light and darkness cycles (Mirmiran et al. 1995, Bernard et al. 2007). The secondary peripheral clock is located in almost each and every tissue such as heart, gastrointestinal tract, lung, liver and kidneys which depends on cues other than light such as metabolites, cytokines, circulating hormones and body temperature. At the cellular level, the circadian rhythm works based on the periodical oscillation of several key proteins with a controlled negative feedback loop. The key clock proteins are the positive regulators, CLOCK (Circadian Locomotor Output Cycles Kaput), BMAL1 (Brain and Muscle Arnt-protein 1, Arnt1) and NPAS2 (Neuronal PAS domain-containing protein 2). The heterodimer CLOCK and BMAL1 proteins translocate into the nucleus to activate the expression of light-inducible (day-phase) genes. These genes are named as clock controlled genes (CCGs) (Fig. 1A). CCGs represent approximately $10 \%$ of all genes at any given point of time (Masri \& Sassone-Corsi 2010). PER and CRY are CCG genes, whose gene products complete the negative feedback loop by heterodimerising and repressing the activity of CLOCK-BMAL1 thereby turning off the CCG's expression. Other CCG genes, ROR and REV-ERB in turn regulate the levels of BMAL1 expression (Fig. 1A) keeping the circadian rhythm intact. Besides the transcriptional control, circadian factors are regulated at post-transcriptional, translational and posttranslational levels.

\section{Cell cycle, chromosomal DNA replication and circadian rhythm: the interconnection}

Mammalian skin, liver, gastrointestinal, immune cells and hematopoietic cells display rhythmicity in DNA synthesis and mitosis (Levi et al. 2007, Dallmann et al. 2016). Eukaryotic chromosomes are equipped with multiple DNA replication origins where the key replication factors are assembled. During late mitosis to early G1 phases of the cell cycle, the sequential binding of ORC (origin recognition complex), together with Cdt1 and Cdc6 facilitate the binding of inactive MCM (2-7) helicase complex marking pre-replication complex (pre-RC) assembly or replication licensing. Approximately $2-3 \mathrm{~h}$ prior to the onset of $S$ phase, at the G1 restriction point (R-point) each cell commits/decides to enter cell cycle (Blagosklonny \& Pardee 2002). When the cells commit to enter cell cycle and proceed to $S$ phase, upon growth factor induction, the inactive MCM helicase is activated by Dbf4-dependent kinase (DDK) and cyclin-dependent kinases (CDKs) triggering the recruitment of several initiation factors such as Cdc45, GINS forming active CMG complex, together with replicative polymerases to initiate DNA synthesis in $S$ phase.

Deregulation in replication licensing and in R-point control, may in part contribute to DNA replication stress induction, chromosome rearrangement and gene amplification observed in many cancer types (McIntosh $\&$ Blow 2012). The mammalian circadian clock genes carefully regulate the cellular transition from $\mathrm{G} 1$ to $S$ phase by regulating the R-point (restriction-point) protein, p21 (Moser et al. 2018). p21 is a potent CDK inhibitor and it is the master regulator of the cell-cycle progression during G1 to $S$ phase (Fig. 1B). It has been shown that the clock gene BMAL1-/ - knock-out mouse liver cells express high levels of p21 (Grechez-Cassiau et al. 2008), the protein (p21) initially identified for its role in promoting cellcycle arrest in the presence of DNA damage. In addition to cell-cycle arrest, the mammalian p21 also mediates p53 dependent and independent cellular senescence pathways (Abbas \& Dutta 2009). Detailed roles of p21 in tumour suppression and its association with p53 in the presence of DNA damage were extensively reviewed previously (El-Deiry 2016). In this section of the review, we examine the dark side of p21 overexpression in p53 deficient cells and discuss how deregulation in clock gene BMAL1 might further fuel genome instability and trigger cancer initiation. Recent examination of p53 deficient pre-cancerous and cancerous lesions revealed that a subset of cancer cells express high levels of p21 together 
A

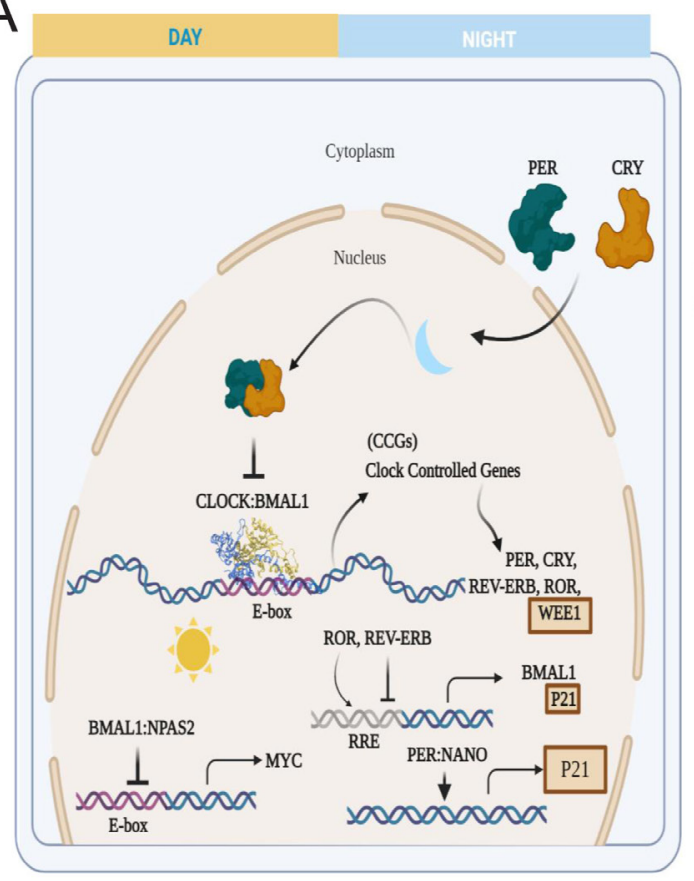

B

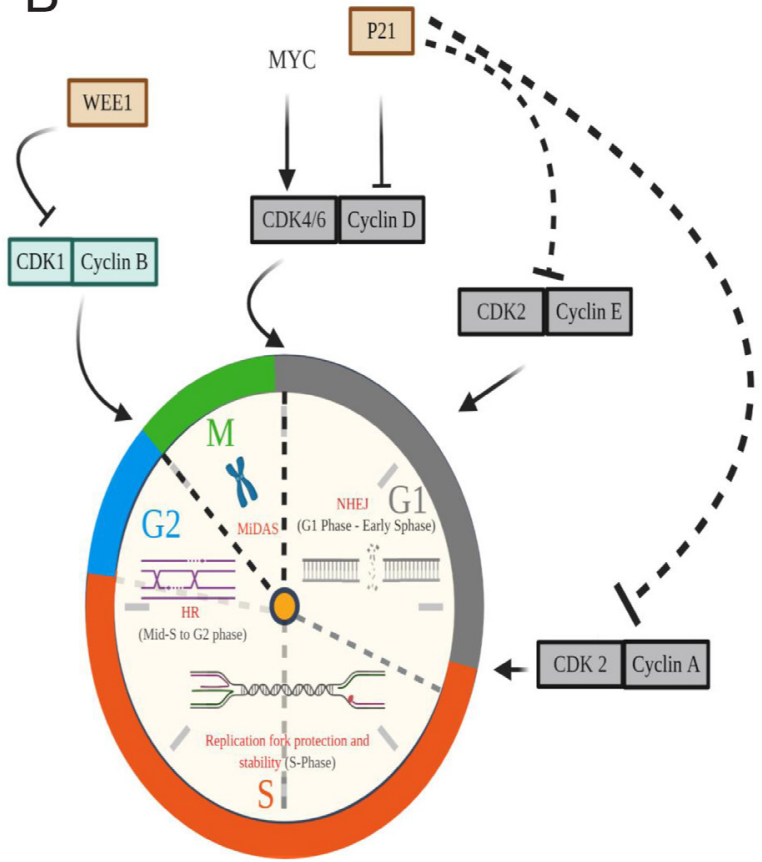

\section{Figure 1}

(A) Circadian rhythm molecular machinery. During day-light, CLOCK:BMAL heterodimer binds onto the E-box to drive expression of circadian clock genes (CCGs). CCG products, PER and CRY are primarily cytoplasmic. During the dark, PER-CRY forms heterodimer inside the nucleus and inhibit BMAL: CLOCK activity to regulate the levels of CCG's completing the negative feedback loop. Similarly, ROR, REV-ERB regulate the level of BMAL expression. ROR, REV-ERB and PER: NANO regulate the level of P21, a cell-cycle inhibitor activated upon P53 activation. BMAL: NPAS2 inhibits the oncogenic stimuli by preventing the expression of MYC. (B) Clock and cell cycle crosstalk: WEE1, P21 regulate cell-cycle phases. For example, WEE1 keeps a check for cells moving from G2 to M phase of the cell cycle and promotes only if HR is complete. P21 is a potent CDK inhibitor activated by P53 that regulates cell-cycle phase and DNA repair choice. In the cell-cycle cartoon, G1 (inset), where NHEJ takes place predominantly, throughout S phase replication fork protection is indispensable, from mid-S to G2 phase of the cell-cycle HR is primarily used to repair DSB's, under-replicated genome has the final chance to repair the chromosome by mitotic DNA Synthesis (miDAS). This figure is created with BioRender.com.

with the proliferation marker Ki-67 (Galanos et al. 2016). Functional insights using inducible p21 overexpression system in p53 null precancerous and cancerous cell lines revealed an oncogenic potential for p21 (Galanos et al. 2016). It has been demonstrated that besides its established role in promoting senescence and cell-cycle arrest, p21 chronic expression in p53 null cells allowed proliferative advantage in a sub-population of cells and these cells apparently escaped senescence (Galanos et al. 2016). Intriguingly, these sub-population of cells exhibited aberrant DNA synthesis and increased genome instability features accompanied with cancer chemoresistance in vitro in the presence of doxorubicin induced double strand breaks (DSBs) (Galanos et al. 2016). Further molecular analyses revealed a close connection between p21 overexpression, p53 status, DNA replication licensing factors and genome instability. Mechanistically, persistent p21 expression prevented the ubiquitin mediated degradation of replication licensing factors Cdc6 and
CDT1 by the E3-ubiquitin ligase complex CRL4-CDT2 (Galanos et al. 2016). This led to the accumulation of replication licensing factors Cdc6 and Cdt 1 that results in unscheduled DNA replication, replication stress induction and proliferative advantage. These results suggest that alterations in p21, both loss- and gain-of-function potentiate cancer cell proliferation depending on cell type and p53 status. On one hand, p21 loss potentiates cell proliferation by allowing cells to pass G1 to S phase prematurely and on the other hand, p21 overexpression in p53 deficient cells allow cells to acquire an aggressive drug-resistant cancer phenotype with increased genome instability features. Overall, evidence suggests that optimal p21 levels are essential to protect normal cells from oncogenic transformation.

Circadian clock gene BMAL1 is altered in several endocrine neoplasms and is the negative regulator of $\mathrm{p} 21$ (Angelousi et al. 2019). It has been observed that BMAL1 expression is significantly upregulated in follicular 


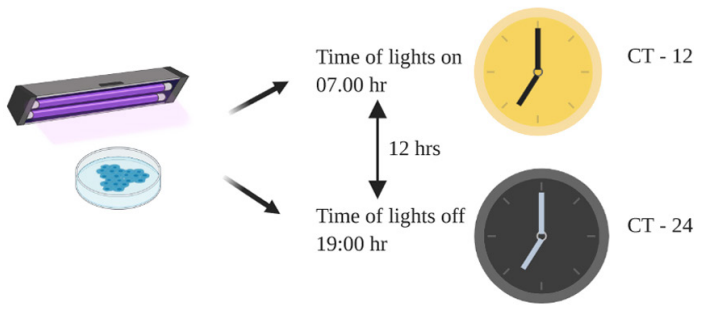

UV irradiation induction

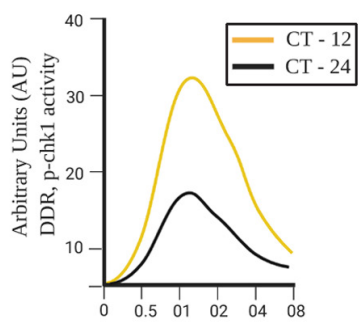

Time (hrs) post

UV

\section{Figure 2}

UV irradiation of mouse fibroblast synchronised to circadian timing. DDR, p-chk1, the read out for DNA damage response (DDR) display rhythmicity in its activity post UV exposure. UV irradiation at CT-12 (In light phase) show two-fold higher DDR activity compared to UV irradiation at CT-24 (in dark phase). This figure is created with BioRender.com.

thyroid carcinoma (FTC) and papillary thyroid carcinoma (PTC) (Mannic et al. 2013). Speculatively, increased BMAL1 expression might perturb the optimal rhythmic expression of p21 leading to restriction point deregulation and premature cell-cycle entry (Grechez-Cassiau et al. 2008). However, in contrast to thyroid cancers, in ovarian cancer cells, BMAL1 is epigenetically silenced by promoter methylation and histone modifications (Yeh et al. 2014). As aforementioned, in this scenario, combination of BMAL1 downregulation and p53 loss may lead to chronic p21 expression, genome instability and cancer initiation due to aberrant DNA synthesis. Largely, these conclusions suggest that the imbalance in BMAL1 might contribute to genome instability in part by deregulating p21 expression levels.

\section{Circadian clock: its link to DNA damage response and repair}

DNA replication stress and DSB's trigger ATR and ATM kinases activity, the key kinases that orchestrate DNA Damage Response (DDR) and downstream DNA repair pathways. Accumulation of ssDNA and subsequent coating of RPA during DNA replication activate ATR kinase that is required for Chk1 phosphorylation (Choi et al. 2010). This activity regulates DNA replication fork speed and is required for the resolution of stalled DNA replication forks (Couch et al. 2013, Toledo et al. 2017). On the other hand, replication stress and DSB's activate ATM kinase to initiate DSB repair pathway such as homologous recombination (HR) and non-homologous end joining (NHEJ). Generally, HR peaks during mid-S to G2 phases of the cell cycle, while NHEJ is active throughout interphase (Hustedt \& Durocher 2016). Recently, hitherto different studies have deciphered the roles of several clock genes like period (PER1, PER2, PER3), timeless (TIM), and cytochrome (CRY1, CRY2) in regulating DDR (Sancar et al. 2010).

(c) 2021 Society for Endocrinology Published by Bioscientifica Ltd. Printed in Great Britain

\section{Rhythmic clock dependent ATR-CHK1 activity}

UV-induced DNA damage primarily produces two types of lesions on the DNA: (1) cylobutane pyrimidine dimers and (2) 6,4-photoproducts (Sancar 2016). These lesions activate ATR-Chk1 DDR signalling pathway to allow nucleotide excision repair (NER) mechanism to function. Recently, it has been shown that, upon UV-induced DNA damage, ATR kinase activity follows a rhythmic pattern to allow downstream circadian NER activity (Kang \& Leem 2014). In mouse fibroblast and liver cells, upon UV induction, p-chk1 and p-p53, a readout for DDR was measured as a function of circadian time. Interestingly, upon UV induction, the DDR, the p-p53 and ATR-Chk1 activity oscillated for a 24-h period displaying two- to three-fold difference between the zenith and nadir time (Kang \& Leem 2014) (Fig. 2). Immunoprecipitation experiments revealed that in the presence of UV-induced DNA damage, clock genes CRY1/TIM form a protein complex with Chk1 and this interaction is required for ATR-Chk1 kinase activation (Kang \& Leem 2014) (Fig. 3). Overall, these

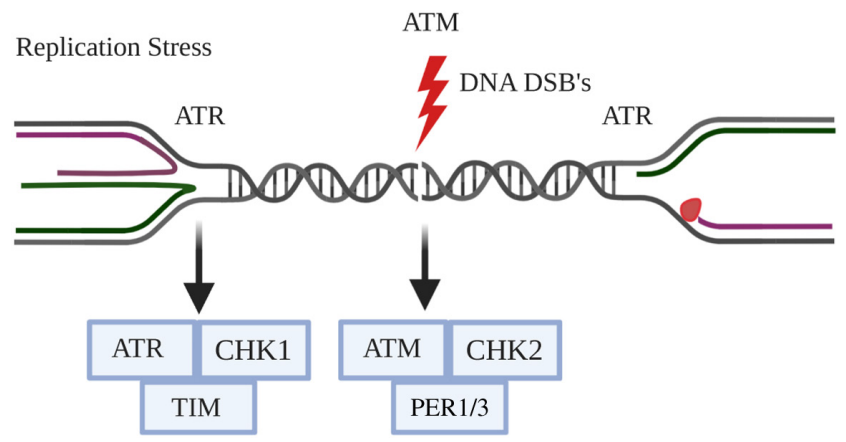

Figure 3

ATR kinase is activated in the presence of replication stress and ATM kinase is activated in the presence of DSBs. ATR kinase interacts physically with timeless one of the CCGs to regulate DNA damage response (DDR). ATM kinase together with its interacting partner Chk2 interacts with PER1/3, a gene required to regulate the expression of CCGs. Note: This figure is just a pictorial representation of different replication/repair intermediates in the replication stress. This figure is created with BioRender.com. 
results suggest that at least in mouse fibroblasts and in liver cells the circadian modulation of DDR Chk1 activity is dependent on clock genes CRY1 and TIM (Fig. 3). In future, it is worth testing whether this rhythmic activity of ATR-Chk1 is also observed in oncogene or reactive oxygen species (ROS) induced replication stress. Cancer cells encountering replication stress constitutively activate ATR, whose selective inhibition by small molecule alters timing of replication initiation, chromatin remodelling coupled with fork elongation defects and S-phase associated cellular lethality (Couch et al. 2013). Holding advantage of ATR inhibition induced cancer cell lethality, different companies developed small molecule inhibitors against ATR and Chk1 that are currently at different phases of clinical trials (Forment \& O'Connor 2018). Further detailed understanding of ATR-Chk1 and its connection with circadian function might provide new approaches to use ATRi for combination cancer therapy.

\section{Clock genes influence ATM-Chk2 kinase activity and DSB repair efficiency}

Clock genes PER 1 and 2 deregulation is associated with initiation and progression of endometrial cancer, breast cancer, colorectal cancer, prostate and head-neck carcinoma (Zheng et al. 2001, Sato et al. 2009). In the presence of induced DSBs, three independent reports confirmed that overexpression of any of the clock genes PER 1 or 2 or 3 sensitised human cancer cells to apoptosis (Gery et al. 2006, Im et al. 2010, Yang et al. 2012). In contrast, downregulation of any of the PER genes independently in cancer cells dampened apoptosis and accelerated cell growth in the presence of DNA damage (Gery et al. 2006, Im et al. 2010, Yang et al. 2012). This suggests a tumour suppressive function for PER genes in response to DNA damage. Upon DSB induction, ATM-Chk2 kinase function promotes reactivation of p53 and induces tumour cell apoptosis (de Lange et al. 2012). Interestingly, all the three PER genes participate in Chk2 activation at least by two distinct pathways to promote apoptosis (Fig. 3) (Gery et al. 2006, Im et al. 2010, Yang et al. 2012). Functionally, upon DSB's induction, PER1/PER3 physically interacts and facilitates CHK2 Thr-68 phosphorylation, whereas PER2 maintains CHK2 Thr-68 phosphorylation status by keeping phosphorylated AKT at low levels (Gery et al. 2006, Im et al. 2010, Yang et al. 2012).

Circadian clock, besides influencing DDR pathway, the daily periodicity of light/darkness (LD) cycle, affects the expression of key DNA repair genes and the efficiency of DSB repair in the presence of ionising radiation (IR) (Palombo et al. 2015). Of note, this DSB repair activity was found to be higher during light phase compared to dark phase (Palombo et al. 2015). While clock gene's role in regulating DDR rhythmicity is evident, landmark studies suggest that DDR in turn affects circadian rhythm and induces circadian phase shifting (Oklejewicz et al. 2008, Gamsby et al. 2009). It was first documented more than 6 decades ago in marine dinoflagellate Gonyaulax polyhedra that the circadian clock can be reset by UV irradiation (Sweeney 1963). In rat fibroblasts, using mPER2 promoterdriven luciferase reporter gene construct, Oklejewicz et al. monitored the real time circadian oscillation in the presence of DNA damage (Oklejewicz et al. 2008). mPER2 promoter activation by BMAL1: CLOCK and its repression by PER/CRY complex indicate the light and darkness phase shifting. Oklejewicz et al. demonstrated a clear phase advancement of bioluminescence rhythm in the presence DSBs, indicating the circadian phase shift (Oklejewicz et al. 2008). Interestingly, these DSBs-induced phase shifting was found to be dependent on ATM mediated damage signalling (Oklejewicz et al. 2008). Likewise, DNA damage generated with either the radiomimetic alkylating drug methyl methanesulfonate (MMS) or UV irradiation in mouse embryonic fibroblasts also found to reset the circadian clock (Gamsby et al. 2009).

Overall, these results discussed from both murine and cultured human cancer cell line models indicate that the circadian clock is intimately intertwined with DDR and DSB repair. In the presence of DNA damage, the clock genes control the rhythmicity of ATR-Chk1 and ATM-Chk2 and this rhythmic DDR response in turn influences circadian phase shifting. Since most of the clock genes discussed above facilitate DDR pathway and are primarily involved in preventing cell proliferation in the presence DNA damage, clock genes are proposed to be tumour suppressor genes. Consistent to its proposed role as tumour suppressor, PER1/2/3 expression was found be significantly low in lung, breast, endometrial pancreatic cancers (Zhang et al. 2020).

\section{Clock gene timeless (TIM) function in DNA replication fork regulation}

Mammalian TIM is originally identified based on its sequence homology with Drosophila melanogaster TIM. Timeless (TIM) physical interaction with other clock genes such as CRY and PER is essential for circadian rhythm generation and maintenance. Independent of its circadian function, TIM is at the heart of molecular pathways 
regulating DDR and DNA repair. Similar to other essential DNA repair genes such as BRCA1, BRCA2, and RAD51, loss of TIM is embryonic lethal (Gotter et al. 2000) and missense driver mutations in human TIM (Q1008E and A429D) predispose to breast cancer (Wood et al. 2007). During $S$ phase of the cell cycle, the replication forks are protected by replication fork protection complex (RPC) to prevent the accumulation of abnormal DNA replication intermediates or DNA breaks (Kolinjivadi et al. 2017). Intriguingly, the clock gene TIM or timeless is a part of RPC which co-purifies with replication proteins such as TIPIN (Errico et al. 2007), CLASPIN and AND1 at the site of replicating nascent-DNA (Somyajit et al. 2017).

Endogenous or exogenous replication stress induced by aldehydes, oncogenes or carcinogens triggers the production of reactive oxygen species (ROS) contributing to genome instability and cancer (Srinivas et al. 2019). The peroxiredoxin (PRDX) family of antioxidant enzymes play an essential function in scavenging ROS and in protecting cells from oxidative DNA damage (De Franceschi et al. 2011). Interestingly, the main ROS scavenging antioxidant-PRDX2 displays a circadian pattern. Their nuclear levels oscillate rhythmically over a period of two entire 24-h cycles (Avitabile et al. 2014). Recently, using single molecular replication assays and biochemical chromatin fractionation techniques, a role for clock gene's TIM and PRDX2 in promoting genome stability in the presence of ROS generation has been proposed (Somyajit et al. 2017). Mechanistically, fluctuation in nucleotide biosynthesis is associated with the generation of reactive oxygen species (ROS), sensed at the replisome by a replisome-based ROS sensor - PRDX2 (Somyajit et al. 2017). When nucleotide biosynthesis falls, activation of PRDX2 by ROS leads to the breakdown of oligomeric form of PRDX2, followed by the eviction of PRDX2 from the replisome along with the fork accelerator - TIMELESS, causing instant fork slow-down (Pai \& Kearsey 2017, Somyajit et al. 2017) (Fig. 4). To preserve genome stability, this reduction in replication fork speed mitigates pathological consequences of replication stress upon ROS induction (Fig. 4). Overall, these results suggest that PRDX2 interact with clock gene TIM to form a replisome-based redox sensor to maintain genome stability (Somyajit et al. 2017).

\section{Circadian clock machinery: intra-adduct DNA lesion removal by nucleotide excision repair (NER)}

Intra-strand and Inter-strand cross link inducing second and third generation cisplatin derivatives are the commonly

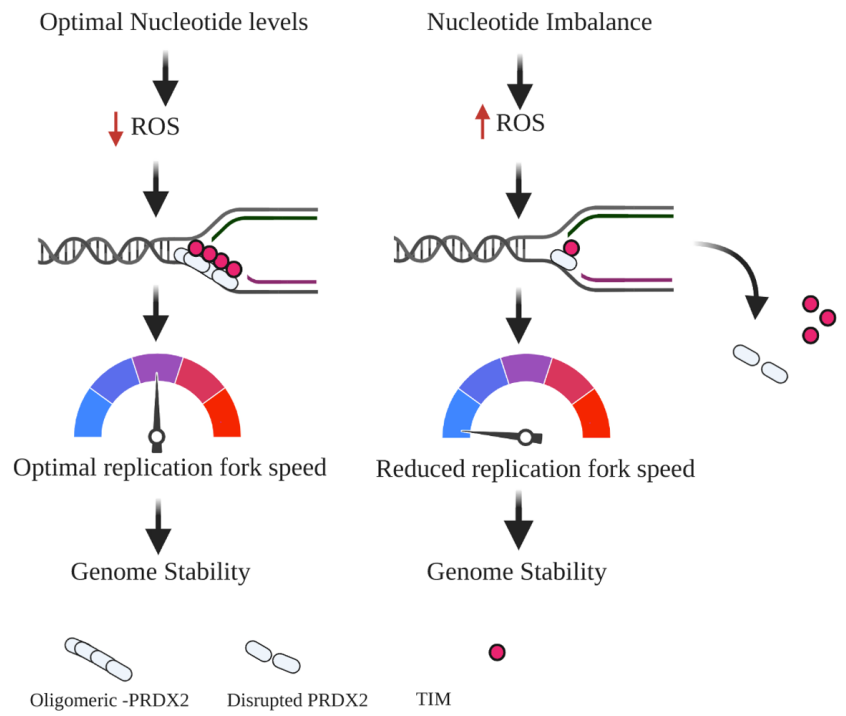

Figure 4

Left: At low levels of ROS, antioxidant oligomeric-PEROXIREDOXIN (PRDX2), a redox-sensor, travels closely with the replisome component together with TIM (timeless), the fork speed accelerator. Right: Alterations in ribonucleotide reductase (RNR) reduce the nucleotide levels triggering reactive oxygen species (ROS). PRDX2 redox sensor's dissociation to smaller sub-units removes its interacting partner TIM from the fork. Eventually fork speed is reduced to prevent unscheduled fork collapse. This figure is created with BioRender.com.

prescribed anti-cancer drugs worldwide (Kelland 2007, Dilruba \& Kalayda 2016). Inter-strand cross link (ICL) lesions occur when Watson-Crick strands are covalently linked preventing transcription and replication reactions, leading to chromosome breaks. The 23 Fanconi Anemia (FA) gene products are required to resolve these ICL lesions (Kolinjivadi et al. 2020). However, predominantly, the DNA lesions induced by cisplatin are intra-strand di-adducts such as Pt-(GpG), Pt-(ApG), and Pt-(GpXpG). In vertebrates, this intra-strand adducts are primarily repaired by nucleotide excision repair (NER) pathway, whose efficiency is critical for chemotherapeutic response. In mammalian cells, at least six essential proteins such as RPA, XPA, XPC, TFIIH, XPG and XPF-ERCC1 are required to complete NER. Together with the single strand binding protein RPA, these structure specific endonucleases remove the lesions in the form of $\sim 24-32$ nt oligomers and the resulting gap is filled by specialised DNA polymerases and ligated (Sancar 1996, Wood 1997, Reardon \& Sancar 2005).

Previous research showed that NER displays tissue specific rhythmicity in DNA repair (Sancar et al. 2010). Using mouse liver and testicular extracts, circadian rhythm of excision repair has been biochemically studied (Kang et al. 2010). Interestingly, it has been demonstrated that the peak excision repair activity was at the circadian timing (c) 2021 Society for Endocrinology Published by Bioscientifica Ltd. Printed in Great Britain 
17:00 $\mathrm{h}$ and lowest repair activity was 05:00 h (Kang et al. 2010). To determine whether this oscillation in DNA repair activity and XPA protein levels are dependent on clock genes, the authors knocked out mouse CRY 1 and 2. Intriguingly, DKO (double knock out) of CRY1 and 2 in mouse abolished the rhythmic expression of XPA and led to persistent accumulation of the XPA protein levels (Kang et al. 2010). Consistent to increased XPA protein levels, the excision repair activity remained constant without demonstrating any circadian pattern (Kang et al. 2010). This suggests that in mouse liver extracts, XPA protein levels and excision repair activity is under the control of CRY1/2 clock genes. Since loss of CRY1 also perturbs rhythmic ATRChk1 activity (Kang \& Leem 2014) (as aforementioned), the impairment in the rhythmicity of excision repair might be due to combination of both compromised rhythmic ATRChk1 activity and XPA protein levels. Further, interactome and biochemical assays revealed that, under the influence of clock genes, HERC2, an ubiquitin ligase interacts with XPA and is responsible for XPA proteolysis. Overall, these results suggest that in addition to DDR control, the excision repair is strongly under the influence of circadian clock machinery in mouse liver. In contrast to liver extracts, excision repair from testicular extracts remained constant throughout $24 \mathrm{~h}$ and did not show any alterations in XPA level or in the excision repair activity (Kang et al. 2010). This suggest that the circadian clock-induced alterations in repair activity might be tissue specific.

Very recently, clock time and excision repair across the genome at single nucleotide resolution have been mapped from mouse liver and kidney cells (Yang et al. 2018). Whole genome circadian repair map revealed that, upon cisplatininduced damage, the repair is controlled by at least two circadian programs (Yang et al. 2018). First, the repair of transcribed strands of the circadian genes is controlled by each gene's phase of transcription, indicating the transcription-coupled DNA repair as an efficient mode of repairing the adducts. This repair falls across the entire circadian cycle with prominent morning and evening circadian peaks (Yang et al. 2018). Secondly, the global repair activity is clock controlled, exhibiting the repair of circadian timing of $\sim 8 \mathrm{~h}$ (Yang et al. 2018). To better understand DNA repair outcome, from clinical perspective, recently, using mouse liver extracts, long-term genomewide kinetic analyses of the effect of circadian rhythm and transcription on cisplatin repair were studied at singlenucleotide resolution (Yang et al. 2019). After injecting cisplatin to mouse, transcribed strand (TS) showed rhythmic repair activity of cisplatin adducts within 2 days. This suggests a transcription-translation feedback loop of circadian clock that exists even after DNA damage response (DDR). However, in contrast, weeks are required to repair non-transcribed strands (NTS) and rest of the genome (Yang et al. 2019). Overall, the results emphasise a circadian clock, transcription- dependent and transcription independent control of DNA repair upon cisplatin treatment. Although most of the studies concluded from mouse liver, brain and kidney extracts, studies from human cells and other tissue types are absolutely required to properly design cisplatin chemotherapy regimens with improved therapeutic outcome. Hence, there is still a great deal of work to be done in this area to improve treatment strategies.

\section{Clock, metabolic alterations and DNA repair}

Over the past decades, the connection between genome instability and metabolite regulation has emerged. Particularly, the changes in metabolite $\mathrm{NAD}^{+}$impact various biological processes such as DNA repair (Ray Chaudhuri \& Nussenzweig 2017), chromatin structure modulation (Hottiger 2015, Posavec Marjanovic et al. 2017), transcription regulation (Kim et al. 2004), telomere function (Doksani \& de Lange 2016), and cell death (Fouquerel \& Sobol 2014), whose deregulation contribute to genome instability (Fig. 5). NAD ${ }^{+}$is the essential cofactor for the enzymatic activity of SIRT1 (NAD+-dependent histone deacetylase sirtuin1) and for DNA repair proteins PARPs in various organisms (Tanny et al. 1999, Imai et al. 2000, Landry et al. 2000, Rosenberg \& Parkhurst 2002, Blander \& Guarente 2004, Hassa \& Hottiger 2008, Canto et al. 2013, Fouquerel \& Sobol 2014) (Fig. 5). Intriguingly, it has been shown that SIRT1 associates with CLOCK, BMAL1 and PER2 in $\mathrm{NAD}^{+}$-dependent manner, to modulate the circadian machinery (Asher et al. 2008, Nakahata et al. 2008). The $\mathrm{NAD}^{+}$level is driven by a ratelimiting enzyme, NAMPT (Nicotinamide phosphoribosyl transferase), which functions in a circadian manner (Nakahata et al. 2009, Ramsey et al. 2009). This periodic $\mathrm{NAD}^{+}$levels impact genome stability via mono- or poly-ADP-ribosylation (PARP family proteins) (Hottiger 2016) and chromatin status via deacetylation (sirtuins) (Chalkiadaki \& Guarente 2015).

\section{PARP1 circadian function: potential chronotherapy for HR-deficient cancers}

For peripheral clocks, feeding-fasting cycles are known to alter circadian rhythm, however, the molecular 


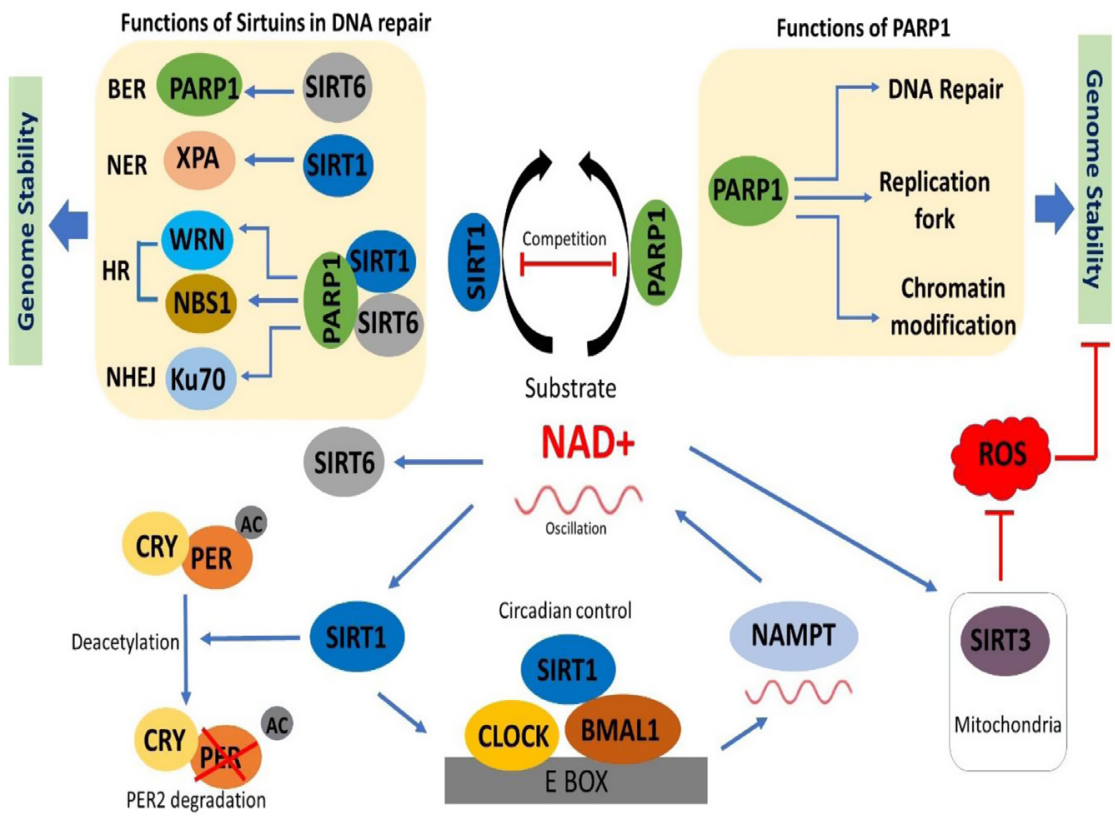

\section{Figure 5}

$\mathrm{NAD}^{+}$is a substrate for PARP enzymes (for mono/ poly-ADP-ribosylation reaction) and sirtuins (for deacetylation reaction). SIRT1 associates with CLOCK: BMAL1 and modulates circadian regulation. SIRT1 controls the stability of clock protein PER2 by promoting deacetylation and leads to PER2 degradation. NAMPT (Nicotinamide phosphoribosyl transferase) gene is regulated by the circadian CLOCK: BMAL1 machinery. NAD ${ }^{+}$ levels are driven by NAMPT (rate-limiting enzyme), both NAD+ and NAMPT oscillate in a circadian manner. PARP1 is known for its functions in DNA repair, stabilisation of DNA replication forks and in the modification of chromatin structure. SIRT1 and SIRT6 regulate DNA repair pathways (Base excision repair (BER), nucleotide excision repair (NER), homologous recombination (HR) and non-homologous end joining (NHEJ)).SIRT6 activates PARP1 in the BER pathway. SIRT1 deacetylates XPA and facilitates the NER pathway. SIRT1 deacetylates the WRN and NBS1, promoting HR. SIRT6 is also recruited to the DSB to promote accumulation of BRCA1 and to deacetylate carboxy-terminal binding protein (CtBP), CtBP-interacting protein (CtIP). In NHEJ pathway, SIRT1 deacetylates KU70 and SIRT6 stabilizes DNA-PK, together enhance NHEJ repair efficiency. Circadian-regulated metabolite $\mathrm{NAD}^{+}$acts as the cofactor for the PARP1 and sirtuins contribute to the genome stability through the participation of PARP1 and/or sirtuins (SIRT1, SIRT6) in DNA repair, replication fork and chromatin modification. SIRT3 (mitochondrial) regulates levels of reactive oxygen species (ROS) influencing genome stability. mechanisms are not well-understood. It was found that the poly-ADP ribosylation (PARylation) activity of PARP1 oscillates with circadian rhythm pattern in mice liver and this PARylation activity is strongly under the influence of nutrient intake (Asher et al. 2010). Further in vivo and in vitro studies demonstrated that PARP1 interacts and PARylates CLOCK: BMAL complex (Asher et al. 2010). In mouse liver cells, this rhythmic PARylation event weakens CLOCK: BMAL DNA binding ability to specific elements such as $\mathrm{E}$ boxes and $\mathrm{G}$ boxes altering the expression levels of CCGs (Asher et al. 2010). Originally, PARP1 is known for its function in base excision repair (BER), replication fork stabilisation and restart of stalled DNA replication forks (Noordermeer \& van Attikum 2019). In the presence of replication stress or DNA damage, PARP1 is rapidly recruited to damage sites and its dissociation requires auto-PARylation through its catalytic activity. It is currently unclear whether this rhythmic function of PARP1 activity is specific to hepatocytes or this might extend to breast, ovarian or prostate tissues. Because mono-allelic germline mutation in error-free (homologous recombination, HR) DNA repair genes such as BRCA1, BRCA2 and Rad51 paralogues are at high risk of developing breast, ovarian or prostate cancers in their lifetime (Venkitaraman 2014). Currently, the first proposed precision medicine PARP1 inhibitors are being used to treat HR-deficient cancers patients (Noordermeer \& van Attikum 2019). Mechanistically, the small molecule PARP1 inhibitors trap PARP1 onto DNA preventing its auto-PARylation or PARylation of target proteins (Murai et al. 2012). This PARP1 trapping induces replication stress and requires HR machinery to repair the broken DNA (Bryant et al. 2005, Maya-Mendoza et al. 2018). Hence, loss-of-function of the HR genes is synthetically lethal with PARP1 inhibition (Kolinjivadi et al. 2017). Clinically, drug resistance for PARP1 inhibitors remains a major obstacle (Noordermeer \& van Attikum 2019). Further understanding of PARP1, NAD ${ }^{+}$and NAMPT's rhythmic activity in breast, ovarian and liver cells may help to treat HR-deficient cancers through chrono-medicine or chronotherapy. Chronotherapy is performed according to the circadian clock pattern to enhance the therapeutic index of a patient and to minimise adverse side effects (Ballesta et al. 2017).
C) 2021 Society for Endocrinology Published by Bioscientifica Ltd. Printed in Great Britain 

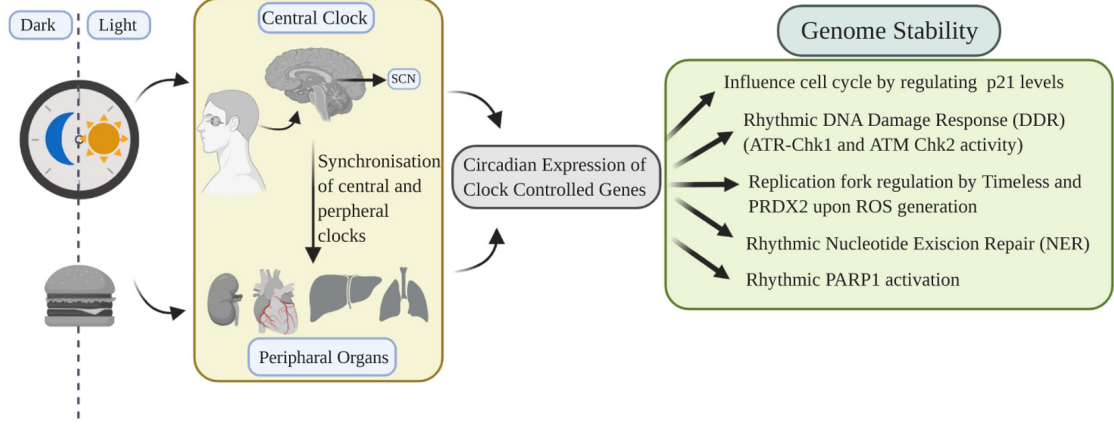

\section{Conclusions}

Evidence from different eukaryotic model systems discussed previously reveals a tight connection between circadian clock, cell-cycle regulation, DDR and DNA repair (Strzyz 2017). To maintain genome stability, circadian clock system influences (1) G1 to S phase transition by regulating p21 levels, (2) maintains the rhythmicity of ATR-Chk1 and ATM-Chk2, DDR activity, (3) alters DNA replication fork velocity in the presence of ROS, (4) maintains rhythmicity of NER and (5) PARP1 activity (Fig. 6). Deregulation in any of these pathways triggers replication stress and alters the DNA repair capacity, leading to genome instability and cancer. Replication stress and DNA breaks are primarily sensed by ATR kinase and PARP1 to stabilise the replication forks and to repair the damaged DNA. Since cancer cells display constitutive replication stress, currently, ATR or PARP1 inhibitors are being tested either as monotherapy or in combination with chemotherapeutic agents (Weber \& Ryan 2015). Initially, the PARP1 inhibitors showed clinical success, but drug resistance to PARP1 inhibitors emerged rapidly(Noordermeer \& van Attikum 2019). Similarly, potential resistance mechanisms for ATR inhibitors have been proposed from the lessons learnt from genetic mouse models and cultured mammalian cell lines (Lecona $\&$ Fernandez-Capetillo 2018). The combination of ATR and PARP inhibitors display synergy and potentiated cancer cell death in many different cancer types (Lecona \& Fernandez-Capetillo 2018). A number of examples discussed previously in this article apparently indicate that circadian genes set the stage for the rhythmicity of ATR-Chk1, ATM-Chk2 and PARP1 activity oscillating downstream repair activity. Rhythmic ATR-Chk1 and PARP1 activity in response to DNA damage hints at a

\section{Figure 6}

Light/darkness and feeding cycles regulate the expression of clock-controlled genes (CCGs). The light sensed by retino-hypothalamic tract is transmitted to suprachiasmatic nucleus (SCN) where the central circadian clock is located. Synchronisation of central and peripheral clocks (organs such as kidney, heart, liver and lung) co-ordinate the expression of clock-controlled genes (CCGs). Clock controlled genes in turn maintain genome stability by regulating optimal p21 levels, maintaining the rhythmicity of DDR activity, regulating replication fork progression in the presence of ROS by timeless gene, maintaining the rhythmicity of NER and PARP1 activation. This figure is created with BioRender.com. possibility of using chrono-therapy for cancer treatment. Modulated chronotherapy for ATR and PARP1 inhibitors might potentially overcome drug resistance and may improve the patient's quality of life partly by reducing adverse side effects.

Though chrono-medicine or chrono-therapy holds substantial promise, the signalling pathways and molecules inter-linking DDR, DNA repair and circadian clock genes are unclear. These challenges warrant ongoing research effort in this area. In order to translate the laboratory findings to clinics, further molecular investigations in a cohort of patient samples are necessary. For example, using the patient-derived organoids will be useful for testing the relationship between clock genes expression, DNA repair capacity and treatment outcome. Since most of the cancer therapy functions based on inhibiting cell proliferation or by inducing DNA break, it is now essential to understand the expression levels of clock-controlled genes, proliferation markers, DNA repair and DDR genes from cancer tissues. The advanced multiplex-immuno histo-chemistry (IHC) might help to stain these multiple factors from the cancer tissues in comparison to normal (Yeong et al. 2020). Correlating the expression levels from different types and stages of cancer will further deepen our understanding about the inter-connection between circadian clock, genome instability and cancer.

\section{Declaration of interest}

The authors declare that there is no conflict of interest that could be perceived as prejudicing the impartiality of this review.

\section{Funding}

This work did not receive any specific grant from any funding agency in the public, commercial or not-for-profit sector.
(C) 2021 Society for Endocrinology Published by Bioscientifica Ltd. Printed in Great Britain 


\section{References}

Abbas T \& Dutta A 2009 p21 in cancer: intricate networks and multiple activities. Nature Reviews: Cancer 9 400-414. (https://doi.org/10.1038/ nrc2657)

Ahuja AK, Jodkowska K, Teloni F, Bizard AH, Zellweger R, Herrador R, Ortega S, Hickson ID, Altmeyer M, Mendez J, et al. 2016 A short G1 phase imposes constitutive replication stress and fork remodelling in mouse embryonic stem cells. Nature Communications 710660. (https://doi.org/10.1038/ncomms10660)

Angelousi A, Kassi E, Ansari-Nasiri N, Randeva H, Kaltsas G \& Chrousos G 2019 Clock genes and cancer development in particular in endocrine tissues. Endocrine-Related Cancer 26 R305-R317. (https:// doi.org/10.1530/ERC-19-0094)

Asher G, Gatfield D, Stratmann M, Reinke H, Dibner C, Kreppel F, Mostoslavsky R, Alt FW \& Schibler U 2008 SIRT1 regulates circadian clock gene expression through PER2 deacetylation. Cell 134 317-328. (https://doi.org/10.1016/j.cell.2008.06.050)

Asher G, Reinke H, Altmeyer M, Gutierrez-Arcelus M, Hottiger MO \& Schibler U 2010 Poly(ADP-ribose) polymerase 1 participates in the phase entrainment of circadian clocks to feeding. Cell 142 943-953. (https://doi.org/10.1016/j.cell.2010.08.016)

Avitabile D, Ranieri D, Nicolussi A, D'Inzeo S, Capriotti AL, Genovese L, Proietti S, Cucina A, Coppa A, Samperi R, et al. 2014 Peroxiredoxin 2 nuclear levels are regulated by circadian clock synchronization in human keratinocytes. International Journal of Biochemistry and Cell Biology 53 24-34. (https://doi.org/10.1016/j.biocel.2014.04.024)

Ballesta A, Innominato PF, Dallmann R, Rand DA \& Levi FA 2017 Systems chronotherapeutics. Pharmacological Reviews 69 161-199. (https://doi.org/10.1124/pr.116.013441)

Bernard S, Gonze D, Cajavec B, Herzel H \& Kramer A 2007 Synchronization induced rhythmicity of circadian oscillators in the suprachiasmatic nucleus. PLoS Computational Biology 3 e68. (https:// doi.org/10.1371/journal.pcbi.0030068)

Blagosklonny MV \& Pardee AB 2002 The restriction point of the cell cycle. Cell Cycle 1 103-110. (https://doi.org/10.4161/cc.1.2.108)

Blander G \& Guarente L 2004 The Sir2 family of protein deacetylases. Annual Review of Biochemistry 73 417-435. (https://doi.org/10.1146/ annurev.biochem.73.011303.073651)

Bryant HE, Schultz N, Thomas HD, Parker KM, Flower D, Lopez E, Kyle S, Meuth M, Curtin NJ \& Helleday T 2005 Specific killing of BRCA2-deficient tumours with inhibitors of poly(ADP-ribose) polymerase. Nature 434 913-917. (https://doi.org/10.1038/ nature03443)

Canto C, Sauve AA \& Bai P 2013 Crosstalk between poly(ADP-ribose) polymerase and sirtuin enzymes. Molecular Aspects of Medicine 34 1168-1201. (https://doi.org/10.1016/j.mam.2013.01.004)

Chalkiadaki A \& Guarente L 2015 The multifaceted functions of sirtuins in cancer. Nature Reviews: Cancer 15 608-624. (https://doi. org/10.1038/nrc3985)

Choi JH, Lindsey-Boltz LA, Kemp M, Mason AC, Wold MS \& Sancar A 2010 Reconstitution of RPA-covered single-stranded DNA-activated ATR-Chk1 signaling. PNAS 107 13660-13665. (https://doi. org/10.1073/pnas.1007856107)

Couch FB, Bansbach CE, Driscoll R, Luzwick JW, Glick GG, Betous R, Carroll CM, Jung SY, Qin J, Cimprich KA, et al. 2013 ATR phosphorylates SMARCAL1 to prevent replication fork collapse. Genes and Development 27 1610-1623. (https://doi.org/10.1101/ $\operatorname{gad} .214080 .113)$

Dallmann R, Okyar A \& Levi F 2016 Dosing-time makes the poison: circadian regulation and pharmacotherapy. Trends in Molecular Medicine 22 430-445. (https://doi.org/10.1016/j. molmed.2016.03.004)

De Franceschi L, Bertoldi M, De Falco L, Santos Franco S, Ronzoni L, Turrini F, Colancecco A, Camaschella C, Cappellini MD \& Iolascon A 2011 Oxidative stress modulates heme synthesis and induces peroxiredoxin- 2 as a novel cytoprotective response in betathalassemic erythropoiesis. Haematologica 96 1595-1604. (https://doi. org/10.3324/haematol.2011.043612)

de Lange J, Verlaan-De Vries M, Teunisse AF \& Jochemsen AG 2012

Chk2 mediates RITA-induced apoptosis. Cell Death and Differentiation 19 980-989. (https://doi.org/10.1038/cdd.2011.182)

Dekens MP, Santoriello C, Vallone D, Grassi G, Whitmore D \& Foulkes NS 2003 Light regulates the cell cycle in zebrafish. Current Biology 13 2051-2057. (https://doi.org/10.1016/j.cub.2003.10.022)

Dilruba S \& Kalayda GV 2016 Platinum-based drugs: past, present and future. Cancer Chemotherapy and Pharmacology 77 1103-1124. (https://doi.org/10.1007/s00280-016-2976-z)

Doksani Y \& de Lange T 2016 Telomere-internal double-strand breaks are repaired by homologous recombination and PARP1/Lig3dependent end-joining. Cell Reports 17 1646-1656. (https://doi. org/10.1016/j.celrep.2016.10.008)

Dong G, Yang Q, Wang Q, Kim YI, Wood TL, Osteryoung KW, Van Oudenaarden A \& Golden SS 2010 Elevated ATPase activity of KaiC applies a circadian checkpoint on cell division in Synechococcus elongatus. Cell 140 529-539. (https://doi.org/10.1016/j. cell.2009.12.042)

El-Deiry WS 2016 p21(WAF1) mediates cell-cycle inhibition, relevant to cancer suppression and therapy. Cancer Research 76 5189-5191. (https://doi.org/10.1158/0008-5472.CAN-16-2055)

Errico A, Costanzo V \& Hunt T 2007 Tipin is required for stalled replication forks to resume DNA replication after removal of aphidicolin in Xenopus egg extracts. PNAS 104 14929-14934. (https://doi.org/10.1073/pnas.0706347104)

Feillet C, Krusche P, Tamanini F, Janssens RC, Downey MJ, Martin P, Teboul M, Saito S, Levi FA, Bretschneider T, et al. 2014 Phase locking and multiple oscillating attractors for the coupled mammalian clock and cell cycle. PNAS 111 9828-9833. (https://doi.org/10.1073/ pnas.1320474111)

Forment JV \& O'Connor MJ 2018 Targeting the replication stress response in cancer. Pharmacology and Therapeutics 188 155-167. (https://doi.org/10.1016/j.pharmthera.2018.03.005)

Fouquerel E \& Sobol RW 2014 ARTD1 (PARP1) activation and NAD(+) in DNA repair and cell death. DNA Repair 23 27-32. (https://doi. org/10.1016/j.dnarep.2014.09.004)

Galanos P, Vougas K, Walter D, Polyzos A, Maya-Mendoza A, Haagensen EJ, Kokkalis A, Roumelioti FM, Gagos S, Tzetis M, et al. 2016 Chronic p53 independent p21 expression causes genomic instability by deregulating replication licensing. Nature Cell Biology 18 777-789. (https://doi.org/10.1038/ncb3378)

Gamsby JJ, Loros JJ \& Dunlap JC 2009 A phylogenetically conserved DNA damage response resets the circadian clock. Journal of Biological Rhythms 24 193-202. (https://doi.org/10.1177/0748730409334748)

Gery S, Komatsu N, Baldjyan L, Yu A, Koo D \& Koeffler HP 2006 The circadian gene per 1 plays an important role in cell growth and DNA damage control in human cancer cells. Molecular Cell 22 375-382. (https://doi.org/10.1016/j.molcel.2006.03.038)

Gotter AL, Manganaro T, Weaver DR, Kolakowski LF, Possidente B, Sriram S, MacLaughlin DT \& Reppert SM 2000 A time-less function for mouse timeless. Nature Neuroscience 3 755-756. (https://doi. org/10.1038/77653)

Grechez-Cassiau A, Rayet B, Guillaumond F, Teboul M \& Delaunay F 2008 The circadian clock component BMAL1 is a critical regulator of p21WAF1/CIP1 expression and hepatocyte proliferation. Journal of Biological Chemistry 283 4535-4542. (https://doi.org/10.1074/jbc. M705576200)

Hassa PO \& Hottiger MO 2008 The diverse biological roles of mammalian PARPS, a small but powerful family of poly-ADP-ribose polymerases. Frontiers in Bioscience 13 3046-3082. (https://doi. org/10.2741/2909)

Hirayama J, Cardone L, Doi M \& Sassone-Corsi P 2005 Common pathways in circadian and cell cycle clocks: light-dependent
(C) 2021 Society for Endocrinology Published by Bioscientifica Ltd. Printed in Great Britain 
activation of Fos/AP-1 in zebrafish controls CRY-1a and WEE-1. PNAS 102 10194-10199. (https://doi.org/10.1073/pnas.0502610102)

Hong CI, Zamborszky J, Baek M, Labiscsak L, Ju K, Lee H, Larrondo LF, Goity A, Chong HS, Belden WJ, et al. 2014 Circadian rhythms synchronize mitosis in Neurospora crassa. PNAS 111 1397-1402. (https://doi.org/10.1073/pnas.1319399111)

Hottiger MO 2015 Nuclear ADP-ribosylation and its role in chromatin plasticity, cell differentiation, and epigenetics. Annual Review of Biochemistry 84 227-263. (https://doi.org/10.1146/annurevbiochem-060614-034506)

Hottiger MO 2016 SnapShot: ADP-ribosylation signaling. Molecular Cell 62 472. (https://doi.org/10.1016/j.molcel.2016.04.019)

Hustedt N \& Durocher D 2016 The control of DNA repair by the cell cycle. Nature Cell Biology 19 1-9. (https://doi.org/10.1038/ncb3452)

Im JS, Jung BH, Kim SE, Lee KH \& Lee JK 2010 Per3, a circadian gene, is required for Chk2 activation in human cells. FEBS Letters $\mathbf{5 8 4}$ 4731-4734. (https://doi.org/10.1016/j.febslet.2010.11.003)

Imai S, Armstrong CM, Kaeberlein M \& Guarente L 2000 Transcriptional silencing and longevity protein Sir2 is an NAD-dependent histone deacetylase. Nature 403 795-800. (https://doi.org/10.1038/35001622)

Kang TH \& Leem SH 2014 Modulation of ATR-mediated DNA damage checkpoint response by cryptochrome 1 . Nucleic Acids Research 42 4427-4434. (https://doi.org/10.1093/nar/gku094)

Kang TH, Lindsey-Boltz LA, Reardon JT \& Sancar A 2010 Circadian control of XPA and excision repair of cisplatin-DNA damage by cryptochrome and HERC2 ubiquitin ligase. PNAS 107 4890-4895. (https://doi.org/10.1073/pnas.0915085107)

Kelland L 2007 The resurgence of platinum-based cancer chemotherapy. Nature Reviews: Cancer 7 573-584. (https://doi.org/10.1038/nrc2167)

Kim MY, Mauro S, Gevry N, Lis JT \& Kraus WL 2004 NAD+-dependent modulation of chromatin structure and transcription by nucleosome binding properties of PARP-1. Cell 119 803-814. (https://doi. org/10.1016/j.cell.2004.11.002)

Kolinjivadi AM, Sannino V, De Antoni A, Techer H, Baldi G \& Costanzo V 2017 Moonlighting at replication forks - a new life for homologous recombination proteins BRCA1, BRCA2 and RAD51. FEBS Letters 591 1083-1100. (https://doi.org/10.1002/1873-3468.12556)

Kolinjivadi AM, Crismani W \& Ngeow J 2020 Emerging functions of fanconi anemia genes in replication fork protection pathways. Human Molecular Genetics 29 R158-R164. (https://doi.org/10.1093/ hmg/ddaa087)

Kowalska E, Ripperger JA, Hoegger DC, Bruegger P, Buch T, Birchler T, Mueller A, Albrecht U, Contaldo C \& Brown SA 2013 NONO couples the circadian clock to the cell cycle. PNAS 110 1592-1599. (https:// doi.org/10.1073/pnas.1213317110)

Landry J, Sutton A, Tafrov ST, Heller RC, Stebbins J, Pillus L \& Sternglanz R 2000 The silencing protein SIR2 and its homologs are NAD-dependent protein deacetylases. PNAS 97 5807-5811. (https:// doi.org/10.1073/pnas.110148297)

Laranjeiro R, Tamai TK, Peyric E, Krusche P, Ott S \& Whitmore D 2013 Cyclin dependent kinase inhibitor p20 controls circadian cell-cycle timing. PNAS 110 6835-6840. (https://doi.org/10.1073/ pnas.1217912110)

Lecona E \& Fernandez-Capetillo O 2018 Targeting ATR in cancer. Nature Reviews: Cancer 18 586-595. (https://doi.org/10.1038/s41568-0180034-3)

Levi F, Filipski E, Iurisci I, Li XM \& Innominato P 2007 Cross-talks between circadian timing system and cell division cycle determine cancer biology and therapeutics. Cold Spring Harbor Symposia on Quantitative Biology 72 465-475. (https://doi.org/10.1101/ sqb.2007.72.030)

Mannic T, Meyer P, Triponez F, Pusztaszeri M, Le Martelot G, Mariani O, Schmitter D, Sage D, Philippe J \& Dibner C 2013 Circadian clock characteristics are altered in human thyroid malignant nodules. Journal of Clinical Endocrinology and Metabolism 98 4446-4456. (https://doi.org/10.1210/jc.2013-2568)
Marchal C, Sima J \& Gilbert DM 2019 Control of DNA replication timing in the 3D genome. Nature Reviews: Molecular Cell Biology 20 721-737. (https://doi.org/10.1038/s41580-019-0162-y)

Masri S \& Sassone-Corsi P 2010 Plasticity and specificity of the circadian epigenome. Nature Neuroscience 13 1324-1329. (https://doi. org/10.1038/nn.2668)

Matsuo T, Yamaguchi S, Mitsui S, Emi A, Shimoda F \& Okamura H 2003 Control mechanism of the circadian clock for timing of cell division in vivo. Science 302 255-259. (https://doi.org/10.1126/ science.1086271)

Maya-Mendoza A, Moudry P, Merchut-Maya JM, Lee M, Strauss R \& Bartek J 2018 High speed of fork progression induces DNA replication stress and genomic instability. Nature 559 279-284. (https://doi.org/10.1038/s41586-018-0261-5)

McIntosh D \& Blow JJ 2012 Dormant origins, the licensing checkpoint, and the response to replicative stresses. Cold Spring Harbor Perspectives in Biology 4 a012955. (https://doi.org/10.1101/cshperspect.a012955)

Mirmiran M, Koster-Van Hoffen GC \& Bos NP 1995 Circadian rhythm generation in the cultured suprachiasmatic nucleus. Brain Research Bulletin 38 275-283. (https://doi.org/10.1016/0361-9230(95)00100-s)

Mori T, Binder B \& Johnson CH 1996 Circadian gating of cell division in cyanobacteria growing with average doubling times of less than 24 hours. PNAS 93 10183-10188. (https://doi.org/10.1073/ pnas.93.19.10183)

Moser J, Miller I, Carter D \& Spencer SL 2018 Control of the restriction point by Rb and p21. PNAS 115 E8219-E8227. (https://doi. org/10.1073/pnas.1722446115)

Murai J, Huang SY, Das BB, Renaud A, Zhang Y, Doroshow JH, Ji J, Takeda S \& Pommier Y 2012 Trapping of PARP1 and PARP2 by clinical PARP inhibitors. Cancer Research 72 5588-5599. (https://doi. org/10.1158/0008-5472.CAN-12-2753)

Nagoshi E, Saini C, Bauer C, Laroche T, Naef F \& Schibler U 2004 Circadian gene expression in individual fibroblasts: cell-autonomous and self-sustained oscillators pass time to daughter cells. Cell 119 693-705. (https://doi.org/10.1016/j.cell.2004.11.015)

Nakahata Y, Kaluzova M, Grimaldi B, Sahar S, Hirayama J, Chen D, Guarente LP \& Sassone-Corsi P 2008 The NAD+-dependent deacetylase SIRT1 modulates CLOCK mediated chromatin remodeling and circadian control. Cell 134 329-340. (https://doi. org/10.1016/j.cell.2008.07.002)

Nakahata Y, Sahar S, Astarita G, Kaluzova M \& Sassone-Corsi P 2009 Circadian control of the $\mathrm{NAD}^{+}$salvage pathway by CLOCK-SIRT1. Science 324 654-657. (https://doi.org/10.1126/science.1170803)

Noordermeer SM \& van Attikum H 2019 PARP inhibitor resistance: a tug-of-war in BRCA-mutated cells. Trends in Cell Biology 29 820-834. (https://doi.org/10.1016/j.tcb.2019.07.008)

Oklejewicz M, Destici E, Tamanini F, Hut RA, Janssens R \& Van Der Horst GT 2008 Phase resetting of the mammalian circadian clock by DNA damage. Current Biology 18 286-291. (https://doi.org/10.1016/j. cub.2008.01.047)

Pai CC \& Kearsey SE 2017 A critical balance: dNTPs and the maintenance of genome stability. Genes 8 57. (https://doi. org/10.3390/genes8020057)

Palombo P, Moreno-Villanueva M \& Mangerich A 2015 Day and night variations in the repair of ionizing-radiation-induced DNA damage in mouse splenocytes. DNA Repair 28 37-47. (https://doi. org/10.1016/j.dnarep.2015.02.002)

Posavec Marjanovic M, Crawford K \& Ahel I 2017 PARP, transcription and chromatin modeling. Seminars in Cell and Developmental Biology 63 102-113. (https://doi.org/10.1016/j.semcdb.2016.09.014)

Ramsey KM, Yoshino J, Brace CS, Abrassart D, Kobayashi Y, Marcheva B, Hong HK, Chong JL, Buhr ED, Lee C, et al. 2009 Circadian clock feedback cycle through NAMPT-mediated $\mathrm{NAD}^{+}$biosynthesis. Science 324 651-654. (https://doi.org/10.1126/science.1171641)

Ray Chaudhuri A \& Nussenzweig A 2017 The multifaceted roles of PARP1 in DNA repair and chromatin remodelling. Nature Reviews: https://erc.bioscientifica.com

https://doi.org/10.1530/ERC-20-0372 (c) 2021 Society for Endocrinology Published by Bioscientifica Ltd. Printed in Great Britain 
Molecular Cell Biology 18 610-621. (https://doi.org/10.1038/ nrm.2017.53)

Reardon JT \& Sancar A 2005 Nucleotide excision repair. Progress in Nucleic Acid Research and Molecular Biology 79 183-235. (https://doi. org/10.1016/S0079-6603(04)79004-2)

Rosenberg MI \& Parkhurst SM 2002 Drosophila Sir2 is required for heterochromatic silencing and by euchromatic hairy/E(Spl) bHLH repressors in segmentation and sex determination. Cell 109 447-458. (https://doi.org/10.1016/s0092-8674(02)00732-8)

Sancar A 1996 DNA excision repair. Annual Review of Biochemistry 65 43-81. (https://doi.org/10.1146/annurev.bi.65.070196.000355)

Sancar A 2016 Mechanisms of DNA repair by photolyase and excision nuclease (nobel lecture). Angewandte Chemie 55 8502-8527. (https:// doi.org/10.1002/anie.201601524)

Sancar A, Lindsey-Boltz LA, Kang TH, Reardon JT, Lee JH \& Ozturk N 2010 Circadian clock control of the cellular response to DNA damage. FEBS Letters 584 2618-2625. (https://doi.org/10.1016/j. febslet.2010.03.017)

Sato F, Nagata C, Liu Y, Suzuki T, Kondo J, Morohashi S, Imaizumi T, Kato Y \& Kijima H 2009 PERIOD1 is an anti-apoptotic factor in human pancreatic and hepatic cancer cells. Journal of Biochemistry 146 833-838. (https://doi.org/10.1093/jb/mvp126)

Somyajit K, Gupta R, Sedlackova H, Neelsen KJ, Ochs F, Rask MB, Choudhary C \& Lukas J 2017 Redox-sensitive alteration of replisome architecture safeguards genome integrity. Science 358 797-802. (https://doi.org/10.1126/science.aao3172)

Srinivas US, Tan BWQ, Vellayappan BA \& Jeyasekharan AD 2019 ROS and the DNA damage response in cancer. Redox Biology 25101084 (https://doi.org/10.1016/j.redox.2018.101084)

Strzyz P 2017 Circadian rhythms: replication keeps the clock ticking. Nature Reviews: Molecular Cell Biology 18 468-469. (https://doi. org/10.1038/nrm.2017.69)

Sweeney BM 1963 Resetting the biological clock in gonyaulax with ultraviolet light. Plant Physiology 38 704-708. (https://doi. org/10.1104/pp.38.6.704)

Tanny JC, Dowd GJ, Huang J, Hilz H \& Moazed D 1999 An enzymatic activity in the yeast Sir2 protein that is essential for gene silencing. Cell 99 735-745. (https://doi.org/10.1016/s0092-8674(00)81671-2)

Toledo L, Neelsen KJ \& Lukas J 2017 Replication catastrophe: when a checkpoint fails because of exhaustion. Molecular Cell 66 735-749. (https://doi.org/10.1016/j.molcel.2017.05.001)

Venkitaraman AR 2014 Cancer suppression by the chromosome custodians, BRCA1 and BRCA2. Science 343 1470-1475. (https://doi. org/10.1126/science.1252230)
Weber AM \& Ryan AJ 2015 ATM and ATR as therapeutic targets in cancer. Pharmacology and Therapeutics 149 124-138. (https://doi org/10.1016/j.pharmthera.2014.12.001)

Wood RD 1997 Nucleotide excision repair in mammalian cells. Journal of Biological Chemistry 272 23465-23468. (https://doi.org/10.1074/ jbc.272.38.23465)

Wood LD, Parsons DW, Jones S, Lin J, Sjoblom T, Leary RJ, Shen D, Boca SM, Barber T, Ptak J, et al. 2007 The genomic landscapes of human breast and colorectal cancers. Science 318 1108-1113. (https://doi.org/10.1126/science.1145720)

Yang X, He X, Yang Z \& Jabbari E 2012 Mammalian PER2 regulates AKT activation and DNA damage response. Biochemistry and Cell Biology 90 675-682. (https://doi.org/10.1139/o2012-025)

Yang Y, Adebali O, Wu G, Selby CP, Chiou YY, Rashid N, Hu J, Hogenesch JB \& Sancar A 2018 Cisplatin-DNA adduct repair of transcribed genes is controlled by two circadian programs in mouse tissues. PNAS 115 E4777-E4785. (https://doi.org/10.1073/ pnas.1804493115)

Yang Y, Liu Z, Selby CP \& Sancar A 2019 Long-term, genome-wide kinetic analysis of the effect of the circadian clock and transcription on the repair of cisplatin-DNA adducts in the mouse liver. Journal of Biological Chemistry 294 11960-11968. (https://doi.org/10.1074/jbc. RA119.009579)

Yeh CM, Shay J, Zeng TC, Chou JL, Huang TH, Lai HC \& Chan MW 2014 Epigenetic silencing of ARNTL, a circadian gene and potential tumor suppressor in ovarian cancer. International Journal of Oncology 45 2101-2107. (https://doi.org/10.3892/ijo.2014.2627)

Yeong J, Tan T, Chow ZL, Cheng Q, Lee B, Seet A, Lim JX, Lim JCT, Ong CCH, Thike AA, et al. 2020 Multiplex immunohistochemistry/ immunofluorescence (mIHC/IF) for PD L1 testing in triple-negative breast cancer: a translational assay compared with conventional IHC. Journal of Clinical Pathology 73 557-562. (https://doi.org/10.1136/ jclinpath-2019-206252)

Zhang J, Lv H, Ji M, Wang Z \& Wu W 2020 Low circadian clock genes expression in cancers: a meta-analysis of its association with clinicopathological features and prognosis. PLOS ONE 15 e0233508. (https://doi.org/10.1371/journal.pone.0233508)

Zheng B, Albrecht U, Kaasik K, Sage M, Lu W, Vaishnav S, Li Q, Sun ZS, Eichele G, Bradley A, et al. 2001 Nonredundant roles of the mPer1 and mPer2 genes in the mammalian circadian clock. Cell $\mathbf{1 0 5}$ 683-694. (https://doi.org/10.1016/s0092-8674(01)00380-4)

Ziv L \& Gothilf Y 2006 Circadian time-keeping during early stages of development. PNAS 103 4146-4151. (https://doi.org/10.1073/ pnas.0600571103)

Received in final form 22 November 2020

Accepted 7 December 2020

Accepted Manuscript published online 11 December 2020 (c) 2021 Society for Endocrinology Published by Bioscientifica Ltd. Printed in Great Britain 